\title{
Principales demandas educativas por evaluar en la formación de profesionales en la Universidad de Artemisa, Cuba
}

Nilda Delgado-Yanes ${ }^{1}$

Universidad de las Ciencias Informáticas - Cuba nildady@uci.cu

Alipio Omar Pérez-Jacinto ${ }^{2}$

Universidad de Artemisa - Cuba

opejota@gmail.com

DOI: https://doi.org/10.21158/2357514x.v8.n1.2020.2634

Cómo citar este artículo: Delgado-Yanes, N.; Pérez-Jacinto, A. O. (2020). Principales demandas educativas por evaluar en la formación de profesionales en la Universidad de Artemisa, Cuba. Revista Virtu@Imente, 8(1), 77-91. DOI: https://doi.org/10.21158/2357514x.v8.n1.2020.2634

Fecha de recepción: 03 de abril 2019

Fecha de aprobación: 29 de abril 2020

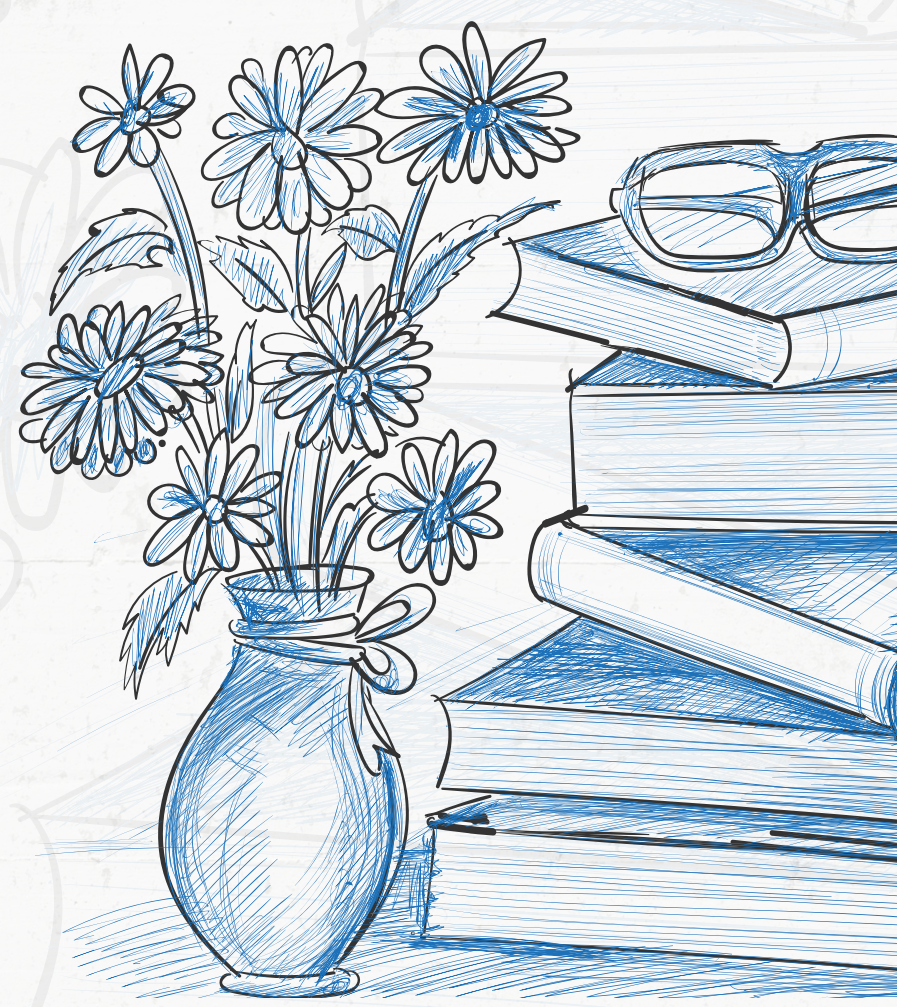

Doctora en Ciencias Pedagógicas. En el área de investigación ha participado en proyectos relacionados con el desarrollo del talento académico y sobre Pedagogía y Didáctica General. Actualmente forma parte del proyecto de investigación "Modelo pedagógico adecuado a las demandas en la formación de profesionales del siglo XXI" de la Universidad de Artemisa. ORCID: https://orcid.org/0000-0002-3617-7719

${ }^{2}$ Doctor en Ciencias Pedagógicas. En el área de investigación ha participado en proyectos relacionados con estudios teóricos sobre la Estadística Inferencial.

ORCID: https://orcid.org/0000-0002-1485-7692 


\section{RESUMEN}

La Universidad de Artemisa se fundó en Cuba en el año 2012, producto de la fusión de varias instituciones de Educación superior con estilos de trabajo diferentes. El proyecto de investigación «Modelo pedagógico adecuado a las demandas en la formación de profesionales del siglo XXl» surge a partir de la necesidad de preparar a su personal docente. Los fundamentos teóricos del proyecto, donde se declaran las demandas educativas del siglo XXI en la formación de profesionales en instituciones de la educación superior, y el diagnóstico del estado inicial de la variable «formación del profesional de pregrado» sirvieron para este trabajo, el cual tiene como objetivo determinar las principales demandas educativas en la formación de profesionales en la Universidad de Artemisa. Los métodos y técnicas empleados fueron: el análisis y síntesis, la deducción-inducción y la matriz Vester, la cual se considera un instrumento de desarrollo que forma parte de la Matriz de Marco Lógico, herramienta analítica utilizada principalmente en la planificación de proyectos de investigación. Se determinaron las demandas educativas de la sociedad cubana del siglo XXI en instituciones de la educación superior, desde lo cognitivo-instrumental y la axiología. Así pues, para la investigación, se analizaron seis demandas educativas y se sometieron a la aplicación de la matriz Vester, con el objetivo de ponderar la prioridad de atención por parte de los profesores. El resultado obtenido sirvió para determinar como principales demandas educativas: las habilidades laborales e investigativas, y el dominio de las Tecnologías de la Información y la Comunicación.

Palabras clave: educación superior; instituciones de educación superior; educación superior en Cuba; educación en Cuba; modelo pedagógico; formación de profesionales. 


\section{Main educational demands to be evaluated in the training of professionals at the University of Artemisa, Cuba}

\section{ABSTRACT}

The University of Artemisa was founded in Cuba in 2012, as a result of the merging of several higher education institutions with different working styles. The research project "Pedagogical model adapted to the training demands of the 21 st Century professionals" arises precisely from the necessity of training its own teaching staff. The theoretical foundations of the project, which not only state the educational demands of the 21st Century regarding the formation of professionals in institutions of higher education, but also include the diagnosis of the initial state of the variable "formation of the undergraduate professional" were used for this work, whose objective is to determine the main educational demands in the training of professionals at the University of Artemisa. The methods and techniques used in this work included: analysis and synthesis, deduction-induction and the Vester matrix, which is considered a development instrument that is part of the Logical Framework Matrix, an analytical tool that is mainly used in the planning of research projects. The educational demands of the Cuban society in the $21 \mathrm{st}$ Century in institutions of higher education were determined from the cognitive-instrumental and axiology aspects. Then, six of those educational demands were analyzed and submitted for the application of the Vester matrix, with the objective of weighing the priority of attention by professors. The results obtained were used to determine the following as the main educational demands: work and research skills, and the mastery of Information and Communication Technologies.

Keywords: higher education; higher education institutions; higher education in Cuba; education in Cuba; pedagogical model; training of professionals. 


\section{Principais demandas educativas por avaliar na formação de profissionais na Universidade de Artemisa, Cuba}

\section{RESUMO}

A Universidade de Artemisa fundou-se em Cuba no ano de 2012, produto da fusão de várias instituições de educação superior com estilos de trabalho diferentes. O projeto de pesquisa "Modelo pedagógico adequado às demandas na formação de profissionais do século XXI" surge a partir da necessidade de preparar seu pessoal docente. Os fundamentos teóricos do projeto, onde se declaram as demandas educativas do século XXI na formação de profissionais em instituições da educação superior, e o diagnóstico do estado inicial da variável "formação do profissional de graduação" serviram para este trabalho, o qual tem como objetivo determinar as principais demandas educativas na formação de profissionais na Universidade de Artemisa. Os métodos e técnicas empregados foram: análise e síntese, dedução-indução e a matriz Vester, a qual se considera um instrumento de desenvolvimento que faz parte da Matriz de Marco Lógico, ferramenta analítica utilizada principalmente no planejamento de projetos de pesquisa. Determinaram-se as demandas educativas da sociedade cubana do século XXI em instituições da educação superior, a partir do cognitivo-instrumental e da axiologia. Por conseguinte, para a pesquisa, analisaram-se seis demandas educativas e submeteram-se ao aplicativo da matriz Vester, com o objetivo de ponderar a prioridade de atenção por parte dos professores. O resultado obtido serviu para determinar como principais demandas educativas: as habilidades trabalhistas e investigativas, e 0 domínio das Tecnologias da Informação e a Comunicação.

Palavras-chave: educação superior; instituições de educação superior; educação superior em Cuba; educação em Cuba; modelo pedagógico; formação de profissionais. 


\section{Principales compétences pédagogiques à évaluer dans la formation des professionnels de l'Université d'Artemisa, Cuba}

\section{RÉSUMÉ}

L'université d'Artemisa a été fondée à Cuba en 2012, fruit de la fusion de plusieurs établissements d'enseignement supérieur aux modes de fonctionement différents. Le projet de recherche «Modèle pédagogique adapté aux exigences de la formation des professionnels au XXle siècle» découle de la nécessité d'une meilleure préparation des enseignants. Ce travail vise à déterminer les principales compétences éducatives de la formation des professionnels de l'Université d'Artemisa et s'appuie sur les fondements théoriques du projet et les exigences éducatives du XXle siècle mises en place dans la formation des professionnels des établissements d'enseignement supérieur avec le diagnostic de l'état initial de la variable «formation des étudiants de premier cycle». Les méthodes utilisées comprennent: l'analyse et la synthèse, la déduction-induction, la matrice de Vester considérée comme un outil d'analyse logique utilisé pour la planification de projets de recherche. . Les exigences éducatives de la société cubaine du XXle siècle et celles des établissements d'enseignement supérieur ont été déterminées selon des aspects cognitivo-instrumentaux et axiologiques. Lors de cette investigation, six compètences pédagogiques ont été analysées et soumises à l'application de la matrice Vester dans le but de déterminer les priorités des enseignants. Les résultats obtenus montrent les principales exigences pédagogiques: compétences investigatives, maîtrise des technologies de l'information et de la communication.

Mots-clés: enseignement supérieur; établissements d'enseignement supérieur; enseignement supérieur à Cuba; éducation à Cuba; modèle pédagogique; formation de professionnels. 


\section{Introducción}

En el primer resultado teórico del proyecto «Modelo pedagógico adecuado a las demandas en la formación de profesionales del siglo XXI», adjunto al programa nacional «Problemas actuales del sistema educativo cubano. Perspectivas de desarrollo», se declara la necesidad emplear un modelo:

Que simplifique el estudio de la realidad y posibilite establecer las bases teóricas, estructurales y funcionales del proceso para que responda a las demandas educativas de la sociedad actual, tanto en el ámbito nacional e internacional, como en el contexto local de la universidad. (Rodríguez, 2017)

A su vez, se determinaron las demandas educativas de la sociedad cubana del siglo XXI en instituciones de la educación superior, desde lo cognitivo-instrumental y la axiología.

El presente trabajo tiene como objetivo: determinar las principales demandas educativas en la formación de profesionales en la Universidad de Artemisa.

\section{La matriz Vester}

Una matriz es un arreglo de filas y columnas donde, por convenio, se ponen las filas de manera horizontal y las columnas de manera vertical. La matriz Vester se utiliza para identificar las causas, los efectos y las relaciones causa-efecto
(Cruz, Pérez, Escalona y Hernández, 2009). En esta matriz, por lo general, se identifican los problemas detectados en el objeto de estudio en análisis previo y se les asigna un número, con el objetivo de evaluar en qué medida cada problema incide en los otros, utilizando para ello, una escala. Luego se procede a ubicar en una tabla, tanto por filas como por columnas en un mismo orden. Además, los problemas se tipifican de acuerdo con su actividad o pasividad en: activos, pasivos, críticos e indiferentes.

El procedimiento para llenar la tabla de la matriz y su posterior interpretación es el siguiente:

a. Seleccionar los problemas más relevantes entre todos los identificados.

b. Asignar una identificación numérica sucesiva a cada problema.

c. Conformar la matriz ubicando los problemas por filas y columnas según el orden asignado.

d. Asignar una valoración del grado de causalidad que merece cada problema con cada uno de los demás, según la escala: 
1. No es causal

2. Causal débil

3. Causal media

4. Causal fuerte

5. Causal muy fuerte

El llenado de la matriz con los valores anteriores obedece al planteamiento: ¿qué grado de causalidad tiene el problema 1 sobre el 2?, ¿sobre el 3?... Y sobre los siguientes, hasta completar cada fila de forma sucesiva y llenar toda la matriz.

Las celdas correspondientes a la diagonal de la matriz quedan vacías, puesto que no se puede relacionar la causalidad de un problema consigo mismo.

La valoración dada a la relación de un problema con el otro se obtiene del consenso entre los criterios del grupo de expertos que está participando.

a) Calcular los totales por filas y columnas. La suma de los totales por filas conduce al total de los activos que se corresponden con la apreciación del grado de causalidad de cada problema sobre los restantes. La suma de cada columna conduce al total de los pasivos que se interpreta como el grado de causalidad de todos los problemas sobre el problema particular analizado, es decir, su nivel como consecuencia o efecto.

b) Para lograr una clasificación de los problemas de acuerdo con las características de causa-efecto de cada uno de ellos, se deben seguir los siguientes pasos:
- Construir un eje de coordenadas en donde en el eje $X$ se sitúen los valores de los activos y en el eje $Y$, el de los pasivos.

- Se suman el valor mayor y el valor menor de activos, y se divide en dos; lo mismo se hace con los pasivos. A partir de los resultados, se trazan sobre los ejes anteriores líneas paralelas al eje $X$, si se trata de los pasivos, $y$ al eje $Y$, si se trata de los activos. Lo anterior facilita un trazado de dos ejes representados por las perpendiculares trazadas desde de los ejes originales, que permite la representación de 4 cuadrantes, ubicando sobre ellos a cada uno de los problemas que se encuentran bajo análisis.

- La ubicación espacial de los problemas en la figura correspondiente facilita la siguiente clasificación:

Cuadrante I (superior derecho): problemas críticos

Cuadrante II (superior izquierdo): problemas pasivos Cuadrante III (inferior izquierdo): problemas indiferentes

Cuadrante IV (inferior derecho): problemas activos 
Tabla 1. Interpretación de cada cuadrante

\begin{tabular}{|c|c|}
\hline $\begin{array}{l}\text { CUADRANTE II: } \\
\text { PASIVOS }\end{array}$ & $\begin{array}{l}\text { CUADRANTE I: } \\
\text { CRÍTICOS }\end{array}$ \\
\hline $\begin{array}{c}\text { Problemas de total pasivos alto } \\
\text { y total activos bajo. } \\
\text { Se entienden como problemas } \\
\text { sin gran influencia causal } \\
\text { sobre los demás, pero que son } \\
\text { causados por la mayoría. } \\
\text { Se utilizan como indicadores } \\
\text { de cambio y de eficiencia de } \\
\text { la intervención de problemas } \\
\text { activos. }\end{array}$ & $\begin{array}{l}\text { Problemas de total activos y } \\
\text { total pasivos alto. } \\
\text { Se entienden como problemas } \\
\text { de gran causalidad que, a } \\
\text { su vez, son causados por la } \\
\text { mayoría de los demás. } \\
\text { Requieren gran cuidado en su } \\
\text { análisis y manejo, ya que de su } \\
\text { intervención dependen en gran } \\
\text { medida los resultados finales. }\end{array}$ \\
\hline $\begin{array}{l}\text { CUADRANTE III: } \\
\text { INDIFERENTES }\end{array}$ & $\begin{array}{l}\text { CUADRANTE IV: } \\
\text { ACTIVOS }\end{array}$ \\
\hline $\begin{array}{l}\text { Problemas de total activos y } \\
\text { total pasivos bajo. } \\
\text { Son problemas de baja } \\
\text { influencia causal, además de } \\
\text { que no son causados por la } \\
\text { mayoría de los demás. } \\
\text { Son problemas de baja } \\
\text { prioridad dentro del sistema } \\
\text { analizado. }\end{array}$ & $\begin{array}{l}\text { Problemas de total activos alto } \\
\text { y total pasivos bajo. } \\
\text { Son problemas de alta } \\
\text { influencia sobre la mayoría de } \\
\text { los restantes, pero que no son } \\
\text { causados por otros. } \\
\text { Son problemas claves, ya } \\
\text { que son causa primaria del } \\
\text { problema central y, por ende, } \\
\text { requieren atención y } \\
\text { manejo crucial. }\end{array}$ \\
\hline
\end{tabular}

Fuente. Elaboración propia.

c) El paso siguiente es jerarquizar los problemas, para lo cual se recomienda, por su sencillez, la técnica de representación en un árbol de problemas.
El árbol identifica un problema central que sirve como pivote para caracterizar a los restantes, según su relación causa-efecto o causa-consecuencia. En función de los resultados de la matriz, el tronco del árbol se forma con el problema más crítico — de más alta puntuación en los activos y pasivos- El resto de los problemas críticos constituyen las causas primarias, mientras que los activos se relacionan con las causas secundarias, y con todas ellas se forman las raíces del árbol. Las ramas del árbol estarán formadas por los problemas pasivos o consecuencias.

- Árbol de objetivos

Se construye a partir del árbol de problemas. El objetivo principal o general se identifica con el problema crítico; los objetivos específicos —-medios-, con las raíces del árbolresto de problemas críticos y activos-, y los resultados esperados, con los problemas pasivos.

- Árbol de alternativas

Se elabora a partir del árbol de objetivos, generando todas las posibles soluciones, vías o caminos para resolver el problema planteado. Estas alternativas son las que pasarán luego al proceso de evaluación más detallado, con el propósito de seleccionar la más adecuada. 


\section{Metodología}

La investigación se desarrolló con un enfoque cuantitativo, para lo cual se recurrió a la aplicación de la matriz Vester como técnica de investigación. La matriz Vester se considera un instrumento de desarrollo que forma parte de la Matriz de Marco Lógico, herramienta analítica utilizada principalmente en la planificación de proyectos de investigación.

En el caso estudiado se analizaron seis demandas educativas de la sociedad cubana del siglo XXI de las propuestas en los fundamentos teóricos del proyecto de investigación «Modelo pedagógico adecuado a las demandas en la formación de profesionales del siglo XX|», en el que se enmarca este trabajo, y se sometieron a la aplicación de la matriz Vester, con el objetivo de ponderar la prioridad de atención por parte de los profesores.

Las demandas educativas de la sociedad cubana del siglo XXI quedan identificadas en la fundamentación teórica del proyecto de investigación y se clasifican en demandas desde lo cognitivo-instrumental y desde lo axiológico, como se detalla a continuación:

Demandas educativas de la sociedad cubana del siglo XXI desde lo cognitivo-instrumental:

- desarrollo del pensamiento crítico;

- independencia para la toma de decisiones;

- autonomía para el aprendizaje a lo largo de toda la vida;
- habilidades laborales e investigativas para la solución creativa de problemas profesionales;

- dominio de las Tecnologías de la Información y la Comunicación (TIC) como fuente y medio de aprendizaje, así como en función del desempeño laboral e investigativo;

- dominio de lenguas extranjeras.

Demandas educativas de la sociedad cubana del siglo XXI desde lo axiológico:

- desarrollo de la responsabilidad profesional, social y laboral;

- solidaridad;

- honestidad;

- dignidad;

- patriotismo;

- humanismo;

- honradez;

- justicia social;

- conciencia de la necesidad del cuidado y conservación del medio ambiente para el desarrollo sostenible;

- respeto a la diversidad.

Luego de establecer los fundamentos teóricos del modelo por presentar, correspondió la caracterización de este en los momentos iniciales de la investigación mediante un diagnóstico del estado de la variable «proceso de formación de pregrado», que se definió conceptualmente como: 
Proceso pedagógico, con énfasis en el proceso de enseñanza aprendizaje en una carrera universitaria, basado en un enfoque teórico-práctico que posibilite la realización de las transformaciones necesarias, para lograr el egreso de profesionales que respondan a las demandas del contexto histórico social del momento. (Rodríguez y Miqueli, 2019, p. 117)

Y su definición operacional se estableció como:

[EI] grado de coherencia del proceso de formación de pregrado a partir de la relación entre la concepción del profesional universitario a formar y el proceso de enseñanza aprendizaje, teniendo en cuenta lo normativo y su concreción práctica, que potencie el egreso de los profesionales que requiere la sociedad. (Rodríguez y Miqueli, 2017, p. 17)

La variable se operacionalizó en dos dimensiones: la teórica-normativa y la práctica. Una de las subdimensiones de la primera es la normativa, que se definió como: «El grado en que los documentos normativos para la formación de pregrado en Cuba evidencian las demandas para la formación de profesionales que requiere la sociedad cubana del siglo $X X \mid »$, y se propuso en un primer análisis con los siguientes documentos:

- modelo del profesional;

- plan del proceso docente;

- programas de disciplinas;

- programas de asignatura;

- objetivos de año académico;

- estrategias curriculares;
- reglamento para el trabajo docente y metodológico en la educación superior;

- reglamento de organización docente de la educación superior, y

- reglamento disciplinario para los estudiantes de la educación superior.

El estado de la dimensión normativa se caracterizó con tres posibles valores —alto, medio o bajo—, según se muestra a continuación:

Alto: las demandas para la formación de profesionales que requiere la sociedad cubana del siglo XXI desde lo cognitivo-instrumental y desde lo axiológico se evidencian en el conjunto de documentos normativos emitidos por el Ministerio de Educación Superior (MES) y que están vigentes para la formación de pregrado.

Medio: las demandas para la formación de profesionales que requiere la sociedad cubana del siglo XXI desde lo cognitivo-instrumental y desde lo axiológico se evidencian parcialmente en el conjunto de documentos normativos emitidos por el MES para la formación de pregrado, pero no faltan las demandas: desarrollo de habilidades laborales e investigativas para la solución de problemas profesionales y dominio de las TIC, como fuente y medio de aprendizaje, o en función del desempeño laboral e investigativo.

Bajo: las demandas para la formación de profesionales que requiere la sociedad cubana del siglo XXI desde lo cognitivoinstrumental y desde lo axiológico no se evidencian en el conjunto de documentos normativos emitidos por el MES para 
la formación de pregrado. Las demandas son: el desarrollo de habilidades laborales e investigativas para la solución de problemas profesionales y el empleo de las TIC, como fuente y medio de aprendizaje, y en función del desempeño laboral e investigativo.

En la evaluación de la subdimensión el desarrollo de habilidades laborales e investigativas para la solución de problemas profesionales, y empleo de las TIC como fuente y medio de aprendizaje, o en función del desempeño laboral e investigativo, se consideran las demandas principales que determinan la evaluación de la subdimensión normativa.

En la confirmación de estas demandas como principales en la evaluación de la dimensión normativa se utilizó la técnica de investigación «matriz Vester».

\section{Resultado de la aplicación de la matriz} Vester en la determinación de las demandas principales en la formación de profesionales en la Universidad de Artemisa

En la aplicación de la matriz en esta investigación se seleccionaron las demandas educativas de la sociedad cubana en la formación de profesionales que se evalúan en el proyecto de investigación «Modelo pedagógico adecuado a las demandas en la formación de profesionales del siglo XX|». Las demandas seleccionadas en la dimensión cognitivo-instrumental son: a) Desarrollo del pensamiento crítico

b) Autonomía para el aprendizaje a lo largo de toda la vida

c) Independencia en la toma de decisiones

d) Dominio de lenguas extranjeras

e) Dominio de las Tecnologías de la Información y la Comunicación como fuente y medio de aprendizaje, así como en función del desempeño laboral e investigativo

f) Habilidades laborales e investigativas para la solución creativa de problemas profesionales

Se procedió a seleccionar las demandas educativas de la sociedad cubana para el siglo XXI que se corresponden con lo cognitivo-instrumental, pues se consideró que en lo axiológico no debe establecerse un nivel jerárquico.

En la evaluación de la relación causal entre las demandas se utilizó la siguiente escala:

1. Sin relación causal

2. Relación causal débil

3. Relación causal media

4. Relación causal fuerte

5. Relación causal muy fuerte

A continuación, se procedió a cruzar las demandas, para lo cual se hizo una matriz como la que se muestra a continuación en la tabla 2: 
Tabla 2. Formato de la matriz

\begin{tabular}{|c|l|l|l|l|l|l|l|}
\hline Demandas & D1 & D2 & D3 & D4 & D5 & D6 & Subtotal EJE X \\
\hline D1 & & & & & & & \\
\hline D2 & & & & & & & \\
\hline D3 & & & & & & & \\
\hline D4 & & & & & & & \\
\hline D5 & & & & & & & \\
\hline D6 & & & & & & & \\
\hline $\begin{array}{c}\text { Subtotal } \\
\text { EJE Y }\end{array}$ & & & & & & & \\
\hline
\end{tabular}

Fuente. Elaboración propia.

El tratamiento que se da a las demandas es el mismo que a los problemas en la explicación inicial sobre la matriz Vester, pues el objetivo es establecer un orden de prioridad entre las demandas, por lo que el procedimiento para llenar la tabla de la matriz y su posterior interpretación es el siguiente:

a) Seleccionar las demandas más relevantes entre las identificadas, que en este caso fueron seis.

b) Asignar una identificación numérica sucesiva a cada demanda, que se correspondió con el orden establecido en la página anterior.

c) Conformar la matriz ubicando las demandas en filas y columnas, de acuerdo con orden asignado. d) Asignar una valoración del grado de causalidad que merece cada demanda con cada una de los demás, según la siguiente escala:

1. Relación no es causal

2. Relación causal débil

3. Relación causal media

4. Relación causal fuerte

5. Relación causal muy fuerte

El llenado de la matriz con los valores anteriores obedece al planteamiento del grado de causalidad que tiene la demanda 1 sobre la 2, sobre la 3 y así sucesivamente, hasta completar cada fila y llenar toda la matriz.

Las celdas correspondientes a la diagonal de la matriz quedan vacías, puesto que no se puede relacionar la causalidad de una matriz consigo misma.

La valoración dada a la relación entre una demanda con la otra se obtiene del consenso entre los criterios del grupo de expertos o especialistas que está participando en la investigación.

e) Calcular los totales por filas y columnas. La suma de los totales por filas conduce al total de las demandas activas que se corresponden con la apreciación del grado de causalidad de cada demanda sobre las restantes. La suma de cada columna conduce al total de las demandas pasivas, que se interpreta como el grado de causalidad de todas las demandas sobre la demanda particular analizada, es decir, su nivel como consecuencia o efecto. 
En la tabla 1, en el eje $X$ están puestas las demandas, del primero al último número, de manera horizontal; en el eje $Y$ van las demandas, del primero al último número, de manera vertical.

A continuación, se procedió a evaluar la influencia de cada demanda en las otras y como resultado se obtuvo la tabla 3, que se muestra a continuación:

Tabla 3. Resultado de la evaluación de las demandas

\begin{tabular}{|c|c|c|c|c|c|c|c|}
\hline Demandas & D1 & D2 & D3 & D4 & D5 & D6 & $\begin{array}{c}\text { Subtotal EJE } \\
\text { X }\end{array}$ \\
\hline D1 & 0 & 2 & 3 & 1 & 5 & 4 & 15 \\
\hline D2 & 1 & 0 & 5 & 2 & 4 & 5 & 17 \\
\hline D3 & 1 & 5 & 0 & 2 & 3 & 5 & 16 \\
\hline D4 & 1 & 1 & 1 & 0 & 5 & 4 & 12 \\
\hline D5 & 4 & 5 & 2 & 3 & 0 & 5 & 19 \\
\hline D6 & 2 & 3 & 5 & 2 & 4 & 0 & 16 \\
\hline $\begin{array}{c}\text { Subtotal } \\
\text { EJE Y }\end{array}$ & 9 & 16 & 16 & 10 & 21 & 23 & \\
\hline
\end{tabular}

Fuente. Elaboración propia.

Luego de evaluar las demandas, se realizó la suma de los valores de las columnas y filas, como se puede apreciar en la tabla 3.
A continuación, se procedió a construir un eje de coordenadas donde en el eje $X$ se situaron los valores de las demandas activas, o sea los que aparecen en las filas, y en el eje $Y$, los de las demandas pasivas, o sea las que aparecen en las columnas.

\section{Figura 1. Demandas activas y pasivas}

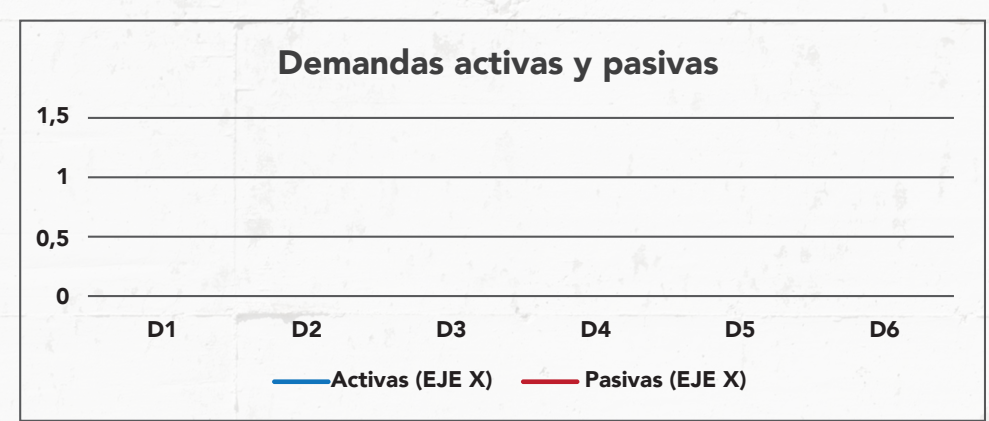

Fuente. Elaboración propia.

Y se determinaron los valores menores y mayores de cada eje, como muestra la tabla 4.

Tabla 4. Identificación de las demandas pasivas y activas

\begin{tabular}{|c|c|c|}
\hline Demandas & Activas (Eje X) & Pasivas (Eje Y) \\
\hline D1 & 15 & 9 \\
\hline D2 & 17 & 16 \\
\hline D3 & 16 & 16 \\
\hline D4 & 12 & 10 \\
\hline D5 & 19 & 21 \\
\hline D6 & 16 & 23 \\
\hline
\end{tabular}

Fuente. Elaboración propia. 
A continuación, se tomaron el valor mayor y el valor menor de las demandas activas, se sumaron y se dividieron entre dos; o sea, 19 más 12, igual a 31, dividido en 2, lo que da un total de 15,5. Lo mismo se hizo con las demandas pasivas: 23 más 9 , igual a 32 , dividido en 2 , lo que da un total de 16 . A partir de los valores resultantes se trazaron sobre los ejes anteriores líneas paralelas al eje $X$ y al eje $Y$. Esto facilitó un trazado de dos ejes representados por las perpendiculares trazadas desde los ejes originales, que permitió la representación de 4 cuadrantes, ubicando sobre ellos a cada uno de las demandas analizadas.

La ubicación espacial de las demandas en la figura correspondiente facilitó la siguiente clasificación:

Cuadrante I (superior derecho): demandas críticas

Cuadrante II (superior izquierdo): demandas pasivas

Cuadrante III (inferior izquierdo): demandas indiferentes

Cuadrante IV (inferior derecho): demandas activas.

Tras obtener el resultado de cada problema en los ejes $X$ y $Y$, se elaboró la gráfica correspondiente, que constó de 4 variables:

- Demandas pasivas -las que pueden tenerse en cuenta, pero en menor cuantía-.

- Demandas críticas -las que influyen más y deben tenerse en cuenta para dar una evaluación entre valores alto, medio y bajo; de al menos medio-.

- Demandas indiferentes —las que pueden despreciarse en un análisis-

- Demandas activas - las que deben tenerse en cuenta en un análisis-
En la figura 2 se muestran:

a) Las demandas pasivas en la parte superior izquierda.

b) Las demandas críticas en la parte superior derecha.

c) Las demandas indiferentes en la parte inferior izquierda

d) Las demandas activas en la parte inferior derecha.

Para elegir el eje Y de la gráfica, se tomó el valor menor en Y y se sumó el valor mayor en Y. Luego, se dividió el resultado entre 2 —en este ejemplo, eje $Y=9+23=32 / 2=16$ -

Para elegir el eje $X$ de la gráfica, se tomó el valor menor en $X$ y se sumó el valor mayor en $X$. Luego, se dividió el resultado entre 2 -en este ejemplo, eje $X=12+19=31 / 2=15,5$ -

El resultado en $X$ y $Y$ se utilizó para marcar la línea de cada eje y determinar el punto centro. El resultado obtenido se muestra en la figura 2:

Figura 2. Ubicación de las demandas en cada cuadrante

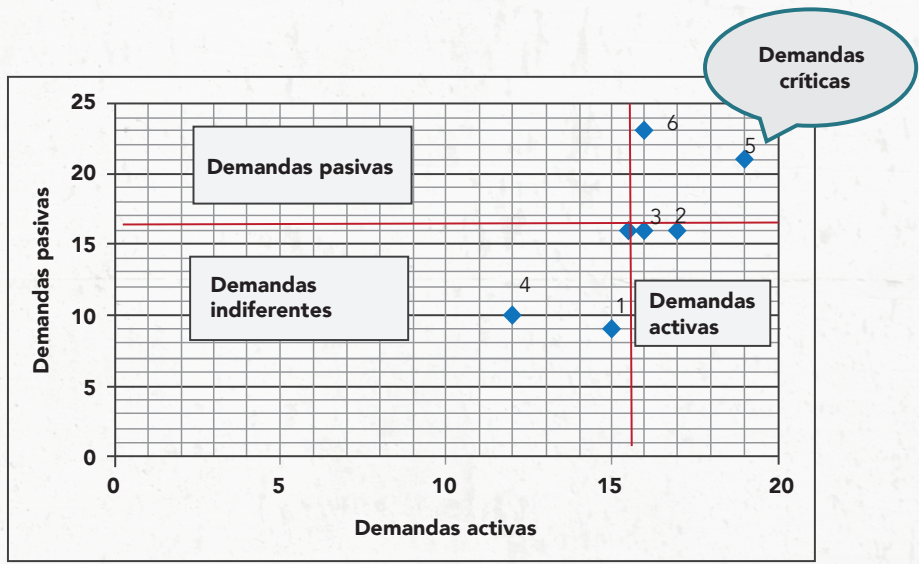

Fuente. Elaboración propia. 
Al analizar la gráfica se puede inferir que las demandas identificadas con los números 5 y 6 son consideradas críticas. Estas son: dominio de las TIC como fuente y medio de aprendizaje, así como en función del desempeño laboral e investigativo, y habilidades laborales e investigativas para la solución creativa de problemas profesionales. Las demandas 2 y 3 son identificadas en el límite entre las variables activas y críticas, estas son: autonomía para el aprendizaje a lo largo de toda la vida e independencia en la toma de decisiones. Las demandas 1 y 4 son indiferentes en la evaluación de la subdimensión; estas son: desarrollo del pensamiento crítico y dominio de lenguas extranjeras.

\section{Conclusiones}

En la etapa de diagnóstico inicial del proyecto de investigación «Modelo pedagógico adecuado a las demandas en la formación de profesionales del siglo $X X \mid »$ se constató el grado en que los documentos normativos vigentes para la formación de pregrado en Cuba ponen en evidencia las demandas educativas que requiere la sociedad cubana del siglo XXI en la formación de profesionales, y para ellos se valoró la posibilidad de considerar las principales demandas.

Tras la aplicación de la técnica de investigación matriz Vester al estudio de las demandas educativas desde lo cognitivo-instrumental, se determinaron las demandas críticas, activas, indiferentes y pasivas. Además, se confirmó la propuesta de considerar principales las siguientes demandas: el dominio de las Tecnologías de la Información y la Comunicación como fuente y medio de aprendizaje, así como en función del desempeño laboral e investigativo, y las habilidades laborales e investigativas para la solución creativa de problemas profesionales, en el estado de la subdimensión normativa de la dimensión teórico-práctica de la variable "formación del profesional de pregrado», ya que constituyen las dos variables críticas del proceso analizado.

\section{Referencias}

Cruz, M.; Pérez, A. O.; Escalona, M.; Hernández, R. (2009). Estadística aplicada a la investigación educativa. La Habana: Educación Cubana. Recuperado de https://bit.ly/3mGfAAl

Rodríguez, A. (2017). Modelo pedagógico para la formación de pregrado en la Universidad de Artemisa: definición, componentes y fundamentos teóricos. Primer resultado de proyecto de investigación. Artemisa, Cuba: Centro de Estudio de Educación y Desarrollo.

Rodríguez, A. y Miqueli, B. (2019). La estructura de la variable proceso de formación de profesores en pregrado. Transformación, 15 (1), $110-128$ 